\title{
吸附 $\mathrm{O}$ 的 $\mathrm{Cu}(110) c(2 \times 1)$ 表面原子结构和电子态 ${ }^{*}$
}

\author{
陈文斌陶向明赵新新蔡建秋谭明秋 \\ (浙江大学物理系, 杭州 310027)
}

\begin{abstract}
摘要 采用第一性原理的密度泛函理论方法计算了清洁 $\mathrm{Cu}(110)$ 表面和吸附 $\mathrm{O}$ 原子的 $\mathrm{Cu}(110) c(2 \times \mathrm{l})$ 表面的原 子结构, 结构弛豫和电子结构, 得到了各种表面结构参数. 分别计算了 $\mathrm{O}$ 原子在 $\mathrm{Cu}(110)$ 表面三个可能吸附位置 吸附后的能量, 并给出了能量最低的吸附位置上各层原子的弛豫特性和态密度. 结果表明 $\mathrm{O}$ 吸附后的 $\mathrm{Cu}(110)$ 表面有附加列(added-row)再构的特性, $\mathrm{O}$ 原子吸附在最表层铜原子上方, 与衬底 $\mathrm{Cu}$ 原子的垂直距离为 0.016 $\mathrm{nm}$, 以氧分子为能量基准的吸附能为 $-1.94 \mathrm{eV}$; 同时由于 $\mathrm{Cu} 3 d-\mathrm{O} 2 p$ 态的杂化作用使得低于费米能级 5.5 6.0 $\mathrm{eV}$ 的范围内出现了局域的表面态. 计算得到清洁的和氧吸附的 $\mathrm{Cu}(110)$ 表面的功函数分别为 $4.51 \mathrm{eV}$ 和 $4.68 \mathrm{eV}$. 电子态密度的结果表明: 在 $\mathrm{Cu}(110) c(2 \times 1)$ 表面 $\mathrm{O}$ 吸附的结构下, 吸附 $\mathrm{O}$ 原子和金属祄底之间的结合主要是由 于最表层 $\mathrm{Cu}$ 原子 $3 d$ 态和 $\mathrm{O}$ 原子 $2 p$ 态的相互作用.
\end{abstract}

关键词： $\mathrm{Cu}(110), \mathrm{O}$ 原子吸附, 附加列再构, 结构弛豫

中图分类号： $\mathrm{O} 641$

近十几年来, 随着计算机和理论技术的不断发 展, 金属表面的模拟计算研究广泛地开展了起来. 由于简单和具有强烈示范意义的特点, $\mathrm{Cu}$ 表面的 条件吸附问题引起了人们极大的兴趣, 其中 $\mathrm{O}$ 吸 附的 $\mathrm{Cu}$ 表面一直是表面科学中重要的研究对象. 研究最多也最热门的是 $c(2 \times 1)$ 结构的表面. 很早以 前人们就发现在室温下 $\mathrm{Cu}(110)$ 表面吸附 $\mathrm{O}$ 时会 发生再构 ${ }^{[1]}$, 但是直到 20 世纪 90 年代, 低能电子 衍射(LEED) $)^{[2]}$ 和 $\mathrm{STM}^{[3]}$ 实验才清楚显示这种再构的 方式是附加列再构. He 束散射 ${ }^{[4]}$, 冲击离子散射谱 (ICISS), X 射线衍射等实验也得到了同样的结果. 在感应 STM 研究中, Coulman 等[3]指出 $(2 \times 1)$ 再构是 由吸附 $\mathrm{O}$ 原子和衬底从原来位置消失的 $\mathrm{Cu}$ 原子之 间的浓缩而形成的. 这个 $c(2 \times 1)$ 再构的生长模式得 到了很多实验的证实 ${ }^{[5-7]}$. 但是以前的关于 $\mathrm{Cu}(110)$ 表面再构的研究是很有限的. 比如有效介质理论给 出的计算结果是成对列再构, 这其实是最低能量的 结果. 所以要得到精确的计算结果, 必须依靠第一性 原理的计算. 事实上, Schimizu 等 ${ }^{[8]}$ 已经采用了从头 计算 $(a b$ initio $)$ 方法来研究 $\mathrm{Cu}(110) c(2 \times 1)-\mathrm{O}$ 附加列 再构, 并用高斯型原子轨道和赝势方法计算了与之 非常相似的 $\mathrm{Ag}(110) c(2 \times 1)-\mathrm{O}$ 再构. 他们指出两者

形成再构机制的不同在于最表层金属原子水平位移 $\Delta$ 的不同, 导致了不同相的再构. 由于 $\mathrm{Ag}(110)$ 表面 上 $\Delta$ 导致的斥力使得其在低覆盖度下 $(N \times 1)$ 相 $(N=7$, $6,5,4,3)$ 比 $(2 \times 1)$ 相更稳定; 而对于 $\mathrm{Cu}(110)$ 面, 由于 其 $\Delta$ 大约为零, 斥力可以忽略, 所以目前只能观测 到 $(2 \times 1)$ 的结构. 尽管如此, 由于结构、参数等原因, 这些工作只是定性地对实验结果做一个验证, 而对 于完整地描述这个吸附进程还是有所欠缺的.

为了从理论上对 $\mathrm{Cu}(110) c(2 \times 1)$ 表面 $\mathrm{O}$ 吸附结 构的物理行为及其 $\mathrm{O}$ 原子的位置进行准确的预言, 解决现有理论计算和实验结果之间的分歧, 我们采 用第一性原理的总能方法, 对上述的吸附表面进行 结构和电子态的计算, 得到了表面结构、吸附结构和 $\mathrm{O}$ 原子位置、表面功函数、电子态密度等系统结果. 计算主要解决了以下问题: (1)计算出了 $c(2 \times 1) \mathrm{O}$ $\mathrm{Cu}(110)$ 表面的结构参数, 确定了表面 $\mathrm{O}$ 原子的位 置和各层 $\mathrm{Cu}$ 原子的弛豫特性; (2)得到了 $\mathrm{O}$ 吸附的 $\mathrm{Cu}(110) c(2 \times 1)$ 表面 $\mathrm{O}$ 原子和各层 $\mathrm{Cu}$ 原子的电子 态密度; (3)讨论了在 $c(2 \times 1)-\mathrm{O}$ 吸附 $\mathrm{Cu}(110)$ 表面结 构下, $\mathrm{O}$ 原子和衬底之间的主要相互作用.

\section{1 计算方法}

2005-03-15 收到初稿, 2005-04-08 收到修改稿. 联系人: 谭明秋(E-mail:mqtan@zju.edu.cn;Tel/Fax: 0571-87951328). “国家自然科学 基金(10204018)资助项目 
密度泛函自洽计算使用了维也纳从头计算模拟

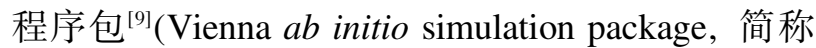
VASP). 这是一个进行第一性原理的量子力学分子 动力学程序包, 采用超软赝势 ${ }^{[10]}$ 和投影子缀加平面 波(projector augmented wave, PAW $)^{[1]}$. 并以平面波为 基函数进行总能和电子结构的计算. 选用了 6 层原 子, 每层含有 2 个共 12 个原子, 即如图 1 中取一个 $(2 \times 1)$ 的格子, 沿缺列方向取 1 个重复单元而垂直缺 列(110)方向取 2 个重复单元来构成 Slab 结构, 其中 五层是基底 $\mathrm{Cu}$ 原子, 另外最上面的一层是表面吸 附的 $\mathrm{O}$ 原子和剩余的 $\mathrm{Cu}$ 原子. 吸附表面另外一边 的三层面是固定的, 作为一个衬底, 而其余的层面则 是可以移动的, 用来模拟原子的弛豫. 我们在刚开始 的时候做了几次比较, 发现取 6 层原子既可以保证 计算结果的精确度, 又不至于使计算量过大. 在两个 表面之外还留有厚度大概 $1 \mathrm{~nm}$ 的真空区域.

计算采用了 $8 \times 16 \times 1$ 的布里渊区 $(\mathrm{BZ})$ 网格, 在由 Monkhors-Pack 方案 ${ }^{[12]}$ 自动产生的不可约 $\kappa$ 点上作 自洽计算. 原子结构优化中的总能收玫判据为 $10^{-5}$ $\mathrm{eV}$, Hellmann-Feynman 原子力的自洽收敛判据为 $10^{-4} \mathrm{Ry} \cdot \mathrm{nm}^{-1}(1 \mathrm{Ry} \approx 13.605 \mathrm{eV})$. 平面波展开的截止 能量为 $300 \mathrm{eV}$, 我们进行过收玫性的检验, 发现设 定这个能量上限足以保证计算的精确度.

\section{2 计算结果及分析}

\section{1 大块铜和清洁的 $\mathrm{Cu}(110)$ 表面弛豫特性}

这里给出大块铜和清洁的 $\mathrm{Cu}(110)$ 表面弛豫特 性的计算分析结果. 其中大块铜的计算中用了 124 个特殊的 $\kappa$ 点, 截止能量为 $292.16 \mathrm{eV}$. 铜的晶格结

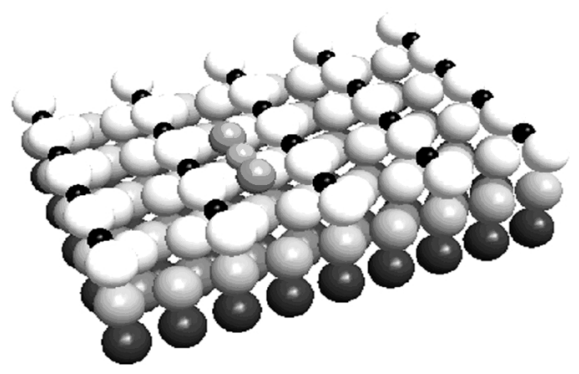

图 $1 \mathrm{Cu}(110) c(2 \times 1)-0$ 表面原子结构示意图

Fig.1 Illustrative atomic structure of $\mathrm{Cu}(110)$ $c(2 \times 1)-0$ surface

The biggest spheres represent copper atoms, the smallest ones are added-row oxygen atoms; the middle size spheres stand for the oxygen atoms adsorbed in the central and side positions.
构参数经过 VASP 代码的优化, 得到其平衡时的晶 格常数为 $a=0.3643 \mathrm{~nm}$, 约比实验值 $0.3615 \mathrm{~nm}^{[13]}$ 大 $0.7 \%$. 一般认为, 这是由于在包含 GGA 交换关联势 ${ }^{[14]}$ 的密度泛函计算中, 低估了 GGA 的修正值造成能 带电子之间的结合被削弱的结果.

$\mathrm{Cu}(110)$ 表面一般情况下不再发生再构, 仅仅有 少许弛豫而已, 即表面层上的原子只在垂直于表面 的方向上移动. 在考虑表面 $\mathrm{O}$ 原子的吸附之前, 先 模拟一下清洁单一的 $\mathrm{Cu}(110)$ 表面. 为了更好地模 拟大块铜的衬底效应, 选取了不同厚度的 $\mathrm{Cu}$ 原子 晶层(Slab)分别计算了它们的表面能、功函数和原子 位置的弛豫特性, 结果发现在取 6 层 Slab 的时候已 经能够达到所需要的精确度. 表 1 中给出了表面弛 豫的计算结果, 已有的理论计算结果、实验值也列在 表 1 中以便比较. 对于 6 层 Slab 的 $\mathrm{Cu}(110)$, 表面和 次表面 $\mathrm{Cu}$ 原子层间的间隔比大块铜晶体中两原子 层间隔(晶格常数)相对收缩了 $10.4 \%$, 这是一个比较 大的弛豫值. 这个结果和 Liem 等 ${ }^{[15]}$ 计算所得的结 果 $10.6 \%$ 非常相近, 比局域密度近似赝势(LDA-PP) 计算 ${ }^{[16]}$ 的值 $9.3 \%$ 要稍大一些, 而各种实验值 ${ }^{[77}$ 比较 离散; 次表层和第三表层的相对膨胀也比较大, 这点 和 $\mathrm{Cu}(100)$ 面有所不同 ${ }^{[18]}$. 除了 $3 、 4$ 层间的弛豫稍有 差异外, 总体来说还是比较相符的. 计算得到层内垂 直表面方向的静电势分布, 与费米能比较后的表面 功函数 $\phi$ 约为 $4.5 \mathrm{leV}$, 与实验值 $(\sim 4.53 \mathrm{~V})$ 符合得相 当好. 得到的清洁 $\mathrm{Cu}(110)$ 表面的表面能为 $9.0 \mathrm{eV}$. $\mathrm{nm}^{-2}$, 而以前我们组计算得到的 $\mathrm{Cu}(100)$ 表面的表面 能为 $8.2 \mathrm{eV} \cdot \mathrm{nm}^{-2}$ [18, 这可能是由于所取的元胞大小、 表面密度、晶格参数和赝势(Uspp 和 Paw)的不同而 造成的. 计算表面能时使用如下的计算步骤:先计算 大块铜中每个铜原子的平均能量 $E_{\text {bulk, }}$, 然后计算整 个包含弛豫的 Slab 的总能量 $E_{\mathrm{slab}}$, 则表面能 $E_{\mathrm{f}}$ 由以 下的公式计算：

$$
E_{\mathrm{f}}=\left(E_{\text {slab }}-N E_{\text {bulk }}\right) / 2 A
$$

其中 $N$ 是 $\mathrm{Slab}$ 中含有的衬底 $\mathrm{Cu}$ 原子的个数, 在我

\section{表 1 清洁 $\mathbf{C u}(110)$ 表面的弛豫情况}

Table 1 Relaxation of clean $\mathrm{Cu}(110)$ surface

\begin{tabular}{cccc}
\hline & $\Delta d_{12} / d_{0}(\%)$ & $\Delta d_{23} / d_{0}(\%)$ & $\Delta d_{34} / d_{0}(\%)$ \\
\hline This Work & -10.4 & +4.6 & -1.2 \\
Liem's Work $^{[15]}$ & -10.6 & +4.9 & +0.4 \\
LDA-PP $^{[16]}$ & -9.3 & +2.8 & +1.1 \\
Experiment $^{[17]}$ & $-3.0 \sim-10.0$ & $+0.0 \sim+8.0$ & \\
\hline$\Delta d_{i j}$ is the variation of distance between two adjacent atomic layers \\
and $d_{0}$ is the layer spacing of two nearest layers in bulk copper
\end{tabular}


们的计算条件下 $N=6, A$ 是一个 Slab 元胞表面的面 积(由于有两个表面, 所以表面积为 $2 A$ ).

在图 2 中还给出了清洁 $\mathrm{Cu}(110)$ 弛豫表面的总 的电子态密度 (DOS). 从上到下依次为最表层 (1st layer), 次表层(2nd layer)和第三表层(3rd layer)Cu 原 子的总态密度. 从图中可以看到表面层能带宽度比 里层的要窄, 这是因为表面层外面没有 $\mathrm{Cu}$ 原子, 使 得它不能向外弛豫, 引起电子分布的适当调整而造成 的. 显然, 表面能级和它的波函数对表面势很敏感.

\subsection{0 吸附的 $\mathrm{Cu}(110) c(2 \times 1)$ 表面原子结构和电} 子态

$\mathrm{O}$ 吸附 $\mathrm{Cu}(110) c(2 \times 1)$ 表面的原子结构示意图 如图 1, 其中最表层由于吸附作用而缺失了中间的 两个 $\mathrm{Cu}$ 原子. 在计算中, 我们从一个没有弛豫的 $\mathrm{Cu}$ 原子薄层出发, $\mathrm{O}$ 原子从 $\mathrm{Cu}$ 原子层所在的位置 开始驰豫, 来确定原子的平衡位置. 我们计算了如图 1 所示的 $\mathrm{O}$ 原子三个可能的吸附位置的能量, 和清 洁的 $\mathrm{Cu}(110)$ 表面计算一样, 我们也是使表层和以 下的两层 $\mathrm{Cu}$ 原子可以运动, 而其余的 $\mathrm{Cu}$ 原子层固 定. 这里我们采用的计算方法和 Cipriani 等 ${ }^{[19]}$ 在计 算 $O$ 原子吸附在 $\operatorname{Ag}(001)$ 表面时采用的方法是一样 的, 且比较所得结果发现也是一致的. 计算发现在中 心位置(center)和边心(site)位置时的 $\mathrm{O}$ 吸附能比附 加列(added-row)位置时的 $\mathrm{O}$ 吸附能都要小, 所以最 稳定的也是最有可能的吸附位置应该是附加列位 置, 计算结果见表 2 . 由此我们可以得出, $\mathrm{Cu}(110)$ 表 面的 $c(2 \times 1)-\mathrm{O}$ 吸附原子结构是附加列再构(addedrow reconstructions).

基于上面 $\mathrm{O}$ 原子吸附的附加列再构的基础上, 我们还计算了它的原子结构, 弛豫特性和电子结构,

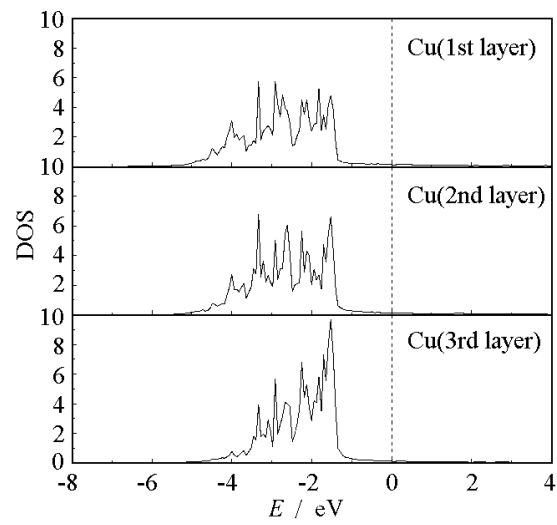

图 2 清洁 $\mathrm{Cu}(110)$ 弛豫表面的电子态密度(DOS)

Fig.2 Density of states(DOS) of relaxed bare $\mathrm{Cu}(110)$ surface
表 $2 \mathrm{Cu}(110) c(2 \times 1)$ 表面 $\mathrm{O}$ 吸附不同位置时的吸附能

Table 2 Adsorption energy $\left(E_{\mathrm{r}}\right)$ of oxygen atom at different adsorption sites on $\mathrm{Cu}(110) c(2 \times 1)$ surface

\begin{tabular}{cc}
\hline adsorption site & $-E_{\mathrm{r}} / \mathrm{eV}$ \\
\hline added-row & 1.94 \\
center & 0.65 \\
side & 0.86 \\
\hline
\end{tabular}

并和 Liem 等 ${ }^{[5]}$, 局域密度近似赝势 $\left(\right.$ LDA-PP) ${ }^{[8]}$, 低 能电子衍射(LEED) $)^{[1]}$, 冲击离子散射谱(ICISS) ${ }^{[5]}, X$ 射线衍射 ${ }^{[20]}$ 等实验的结果做了比较, 见表 3 . 为了确 定 $\mathrm{O}$ 原子和最表层 $\mathrm{Cu}$ 原子之间的位置关系, 先假 定 $\mathrm{O}$ 原子位于最表层和次表层 $\mathrm{Cu}$ 原子之间,计算 结果发现 $\mathrm{O}$ 原子弛豫到了最表层 $\mathrm{Cu}$ 原子外, 而且 弛豫度比上面的计算结果更大一些, 说明我们最初 假设 $\mathrm{O}$ 原子在最外层是正确的. 实际上由于测定 $\mathrm{O}$ 原子之类的轻原子的时候会遇到各种问题, 所以这 个位置是很难确定的(如用 ICISS, X-ray 等方法), 目 前最可靠的结果是用 LEED 测量所得的, 可以看到 我们的结果与之符合得相当好. 从中我们还发现第 三原子层上的两个原子并不处于同一高度, 而次表 层原子高度没有分离, 可以看出次表层的两个原子 实际上在晶格中位置相当, 而第三层两原子则是不 同的. 表面功函数 $\phi$ 约为 $4.68 \mathrm{eV}$, 与清洁 $\mathrm{Cu}(110)$ 表面的功函数相比略高一点.

图 3 给出了 $\mathrm{O}$ 吸附 $\mathrm{Cu}(110) c(2 \times 1)$ 表面附加列 再构后各层原子总的电子态密度 $(\mathrm{DOS})$ 及 $\mathrm{O}$ 原子 $2 p$ 态、最表层 $\mathrm{Cu}$ 原子 $3 d$ 态的分态密度. 注意到次表 层和第三层分别有两种不同的 $\mathrm{Cu}$ 原子态密度, 而 最表层只有一种 $\mathrm{Cu}$ 原子的态密度, 这是因为最表 层的 $\mathrm{O}$ 原子吸附后的附加列再构使得原来的 $\mathrm{Cu}$ 原

\section{表 3 附加列再构后表面各层原子的结构及其弛豫情况}

Table 3 Calculated structure and relaxation parameters of surface layers in the added-row reconstruction configuration (in nm)

\begin{tabular}{lccccc}
\hline & $d_{0}$ & $\Delta d_{12}$ & $\Delta d_{23}$ & $\Delta d_{2}$ & $\Delta d_{3}$ \\
\hline This Work & 0.016 & 0.139 & 0.128 & 0.003 & 0.0023 \\
Liem's Work $^{[15]}$ & 0.020 & 0.141 & 0.128 & 0.002 & \\
LDA-PP $^{[8]}$ & 0.055 & 0.132 & 0.097 & 0.000 & \\
LEED $^{[1]}$ & 0.004 & 0.149 & 0.121 & 0.003 & \\
ICISS $^{[5]}$ & -0.008 & 0.151 & 0.128 & 0.012 & \\
X-rayy $^{[20]}$ & -0.034 & 0.165 & 0.128 & 0.003 & \\
\hline
\end{tabular}

$d_{0}$ is the perpendicular distance between oxygen atom and surface $\mathrm{Cu}$ layer, and $\Delta d_{i j}$ is the distance variation of the $i$ and $j$ surface $\mathrm{Cu}$ atomic layers. $\Delta d_{2}$ is the parallel relaxation of the sublayer $\mathrm{Cu}$ atoms, $\Delta d_{3}$ is the perpendicular relaxation of the third $\mathrm{Cu}$ layer. 

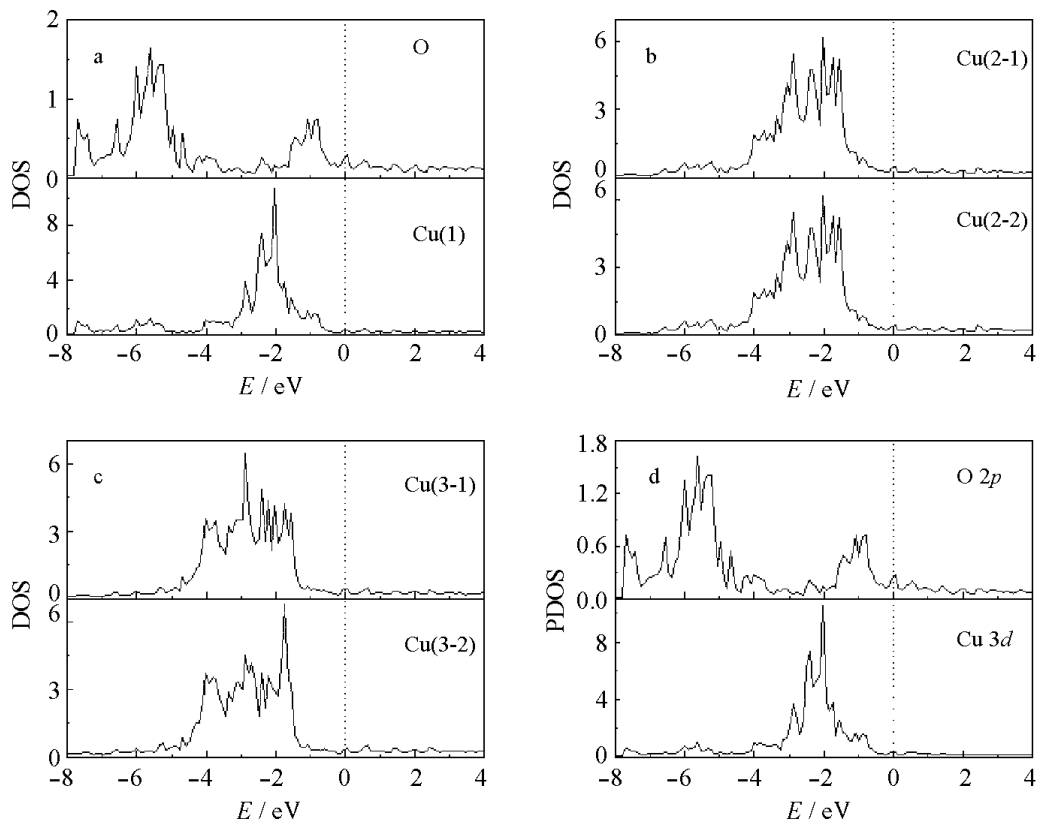

图 30 吸附 $\mathrm{Cu}(110) c(2 \times 1)$ 表面附加列再构后的电子态密度(DOS)

Fig.3 Density of states(DOS) of oxygen-adsorbed $\mathrm{Cu}(110) c(2 \times 1)$ surface in the added-row reconstruction

(a) DOS of $\mathrm{O}$ atom and the first surface $\mathrm{Cu}$ atom, (b) DOS of the second surface $\mathrm{Cu}$ atom, (c) DOS of the third $\mathrm{Cu}$ layer,

(d) partial DOS of $\mathrm{O} 2 p$ and $\mathrm{Cu} 3 d$ states near the surface

子缺失的缘故. 而次表层的两种 $\mathrm{Cu}$ 原子态密度其 实是一样的, 第三层则不同, 这又佐证了我们上面所 说的次表层的两个原子实际上在晶格中位置相当, 而第三层两原子则不同. 我们发现 O 原子也呈现出 一定的金属性, 通过与衬底 $\mathrm{Cu}$ 原子的杂化作用形 成了具有二维特征的 $O$ 的能带. 另外我们还可以看 到, $\mathrm{O}$ 原子的电子态密度在低于费米能级 $4 \mathrm{eV}$ 的范 围内出现了一些态密度峰, 而最表层和次表层的 $\mathrm{Cu}$ 原子态密度在 5.5 6.0 eV 的范围内也有一些峰, 这 在清洁的 $\mathrm{Cu}(110)$ 表面是观察不到的, 而以下各层 在此位置均未观察到态密度峰, 于是可以说明 $\mathrm{O}$ 原 子和最表层、次表层的 $\mathrm{Cu}$ 原子发生了杂化作用. 图 $3 \mathrm{~d}$ 是 $\mathrm{O}$ 原子 $2 p$ 态和最表层 $\mathrm{Cu}$ 原子 $3 d$ 态的分态密 度, 和图 $3 \mathrm{a}$ 比较可以说明, 在 $\mathrm{O}$ 吸附 $\mathrm{Cu}(110) c(2 \times \mathrm{l})$ 表面结构下, $\mathrm{O}$ 原子和衬底之间的结合主要是最表 层和次表层 $\mathrm{Cu} 3 d$ 和 $\mathrm{O} 2 p$ 态的相互作用, 其中最表 层 $\mathrm{Cu}$ 原子占了绝大部分.

\section{3 讨论及结论}

用密度泛函理论和分子动力学方法计算研究了 清洁的 $\mathrm{Cu}(110)$ 和吸附氧的 $\mathrm{Cu}(110) c(2 \times \mathrm{l})$ 表面的原 子结构, 得到了 $\mathrm{O}$ 原子的吸附位置、各表面层 $\mathrm{Cu}$ 原 子的弛豫特性和电子态密度, 从理论上对这一长期
争议的表面吸附问题给出了比较完整精确的计算结 果, 与实验所得结果符合得很好.

从计算分析结果可以看到, 清洁的 $\mathrm{Cu}(110)$ 表 面 $\mathrm{Cu}$ 原子会发生弛豫, 最表层和次表层的 $\mathrm{Cu}$ 原子 相对收缩, 而次表层与第三表层 $\mathrm{Cu}$ 原子则相对膨 胀, 这和理论及各种实验的结果都比较吻合. 计算还 发现 $\mathrm{O}$ 吸附到 $\mathrm{Cu}(110) c(2 \times 1)$ 表面后出现了附加列 再构的表面特征, 其表面吸附能以氧原子作为能量 参考点的值为 $-1.94 \mathrm{eV}$, 氧吸附在中心位置和边位 的吸附能分别为 $-0.65 \mathrm{eV}$ 和 $-0.86 \mathrm{eV}$, 远小于附加列 再构位置的吸附能. 从计算得到的表面原子的电子 态密度中发现, $\mathrm{O}$ 原子呈现出一定的金属性, 而表层 的 $\mathrm{Cu}$ 原子出现了和 $\mathrm{O}$ 原子相关的一些态密度峰, 可 以认为 $\mathrm{O}$ 原子和金属衬底之间的结合主要是最表层 和次表层 $\mathrm{Cu} 3 d$ 和 $\mathrm{O} 2 p$ 态的相互作用一一杂化.

另外, 还通过结构优化得到了该吸附表面的原 子结构参数. 结果说明 $\mathrm{O}$ 吸附的 $\mathrm{Cu}(110)$ 面中 $\mathrm{O}$ 原 子都在 $\mathrm{Cu}$ 原子之上, 和我们组以前的 $\mathrm{Cu}(100)$ 面的 计算结果一致, 可以认为有共性特征. 而 ICISS ${ }^{[5]} 、 \mathrm{X}$ ray ${ }^{[20]}$ 等实验指出, 在附加列再构的图像下, $\mathrm{O}$ 原子 的位置可能位于最表面 $\mathrm{Cu}$ 原子的下面. 为验证此 观点, 用起始的 $\mathrm{O}$ 原子放置在表面 $\mathrm{Cu}$ 原子之下的 设置进行结构的优化计算, 结果 $\mathrm{O}$ 原子还是跑到最 
外层 $\mathrm{Cu}$ 原子以上, 说明 $\mathrm{O}$ 原子在 $\mathrm{Cu}$ 原子之下的可 能并不存在. 我们得到的附加列再构的结果和以前 的采用超软㕍势的计算结果 ${ }^{[15]}$ 符合得相当好, 而实 验中得到的结果则比较离散, 特别是上述 ICISS、Xray 等方法得到的关于 $\mathrm{O}$ 原子位置的结果. 局域密 度近似赝势(LDA-PP)的结果分歧较大, 我们认为这 主要是因为 LDA-PP 方法所采用的范数守恒赝势和 我们目前使用的超软赝势不同. 一般认为, 对于过渡 金属和元素周期表中的第一行元素, 范数守恒噟势 计算得到的结果是不可靠的. 范数守恒条件要求核 内的总噟电荷必须完全等于全电子波函数的电荷, 因此对于 $\mathrm{O} 2 p$ 和 $\mathrm{Cu} 3 d$ 这样复杂的原子轨道, 在一 种条件下得到的赝势不具有一般的传递性, 即使用 于各种化学环境的能力. 超软赝势方法采用几个可 分离项求和的形式, 同时放宽了范数守恒的约束条 件, 使得赝波函数更平滑优化, 并且在核外变得局域 化, 其传递性得到了很大的增强.

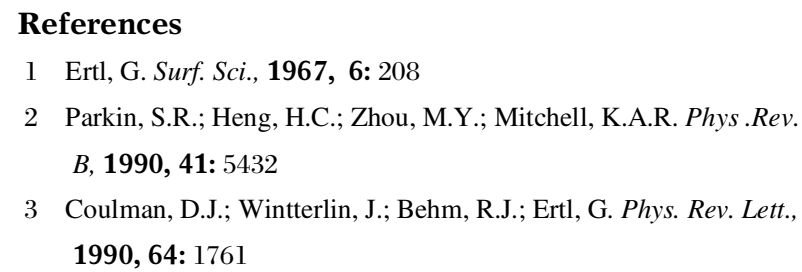

\section{References}

1 Ertl, G. Surf. Sci., 1967, 6: 208

2 Parkin, S.R.; Heng, H.C.; Zhou, M.Y.; Mitchell, K.A.R. Phys .Rev. $B$, 1990, 41: 5432

3 Coulman, D.J.; Wintterlin, J.; Behm, R.J.; Ertl, G. Phys. Rev. Lett., 1990, 64: 1761

4 Lapujoulade, J.; Le Cruer, Y.; Lefort, M.; Lejay, Y. Surf. Sci., 1982, 118: 103

5 Durr, H.; Fauster, Th.; Schneider, R. Surf. Sci., 1991, 244: 237

6 Buisset, J.; Rust, H.-P.; Schweizer, E.K.; Cramer, L.; Bradshaw, A. M. Surf. Sci., 1996, 349: L147

7 Jensen, F.; Besenbacher, F.; Laegsgaard, E.; Stensgaard, I. Phys. Rev. B, 1990, 41: 10233

8 Schimizu, T.; Tsukada, M. Surf. Sci., 1993, 295: 1017

9 Kresse, G.; Furthermuller, J. Comput. Mater. Sci., 1996, 6: 15; Phys. Rev. B, 1996, 54: 11169

10 Vanderbilt, D. Phys. Rev. B, 1990, 41: 7892

11 Blöchl, P.E. Phys. Rev. B, 1994, 50: 17953

12 Monkhorst, H.J.; Pack, J.D. Phys. Rev. B, 1976, 13: 5188

13 Kittel, C. Introduction to solid state physics, 5th ed., New York: John Wiley \& Sons, Inc., 1976

14 Perdew, J.P.; Chevary, J.A.; Vosko, S.H.; Jachson, K.A.; Pederson, M.R.; Singh, D.J.; Fiolhais, C. Phys. Rev. B, 1992, 46: 6671

15 Liem, S.Y.; Kresse, G.; Clarke, J.H.R. Surf. Sci., 1998, 415: 194

16 Rodach, Th.; Bohnen, K.P.; Ho, K.M. Surf. Sci., 1993, 286: 66

17 Adams, D.L.; Nielsen, H.B.; Anderson, J.N. Surf. Sci., 1983, 128: 294

18 Cai, J.Q. Master Dissertation. Hangzhou: Zhejiang University, 2005 [蔡建秋. 硕士学位论文. 杭州: 浙江大学, 2005]

19 Clipriani, G.; Loffreda, D.; Corso, A.D.; Gironcoli, S.; Baroni, S. Surf. Sci., 2002, 501: 182

20 Feidenhans'1, R.; Grey, F.; Johnson, R.L.; Mochrei, S.G.J.; Bohr, J.; Nielsen, M. Phys. Rev. B, 1990, 41: 5420

\title{
The Atomic Structure and Electronic States of Oxygen-adsorbed on $\mathrm{Cu}(110) c(2 \times 1)$ Surface*
}

CHEN, Wen-Bin TAO, Xiang-Ming ZHAO, Xin-Xin CAI, Jian-Qiu TAN, Ming-Qiu

(Department of Physics, Zhejiang University, Hangzhou 310027)

\begin{abstract}
The surface atomic geometry, structural relaxations, and electronic states of clean $\mathrm{Cu}(110)$ surface and oxygen-adsorbed $\mathrm{Cu}(110) c(2 \times 1)$ surface have been studied by using ab initio total energy calculations. The density functional calculations have been carried out for three possible adsorbed positions of oxygen and the most favorable one has been determined by total energy comparison. It reveals that the added-row reconstruction is the most stable one with maximum adsorption energy in $\mathrm{Cu}(110) c(2 \times 1)$ surface and the adsorbed oxygen atom is beyond the outmost surface $\mathrm{Cu}$ layer slightly. The adsorbates lie approximately $0.016 \mathrm{~nm}$ above the outermost $\mathrm{Cu}$ layer and the hybridized band derived from $\mathrm{Cu} 3 d-\mathrm{O} 2 p$ hybridization locates in the range of $-5.5 \sim-6.0 \mathrm{eV}$ below Fermi energy. The adsorption energy of oxygen in this configuration is determined to be $-1.94 \mathrm{eV}$ with respect to oxygen molecule. The work functions of clean $\mathrm{Cu}(110)$ and oxygen-adsorbed $\mathrm{Cu}(110) c(2 \times 1)$ surface are calculated to be $4.51 \mathrm{eV}$ and $4.68 \mathrm{eV}$, respectively. The surface electronic structures show that the cohesive effect between adsorbates and the substrate $\mathrm{Cu}$ atoms is essentially due to the $\mathrm{Cu} 3 d-\mathrm{O} 2 p$ interaction.
\end{abstract}

Keywords: $\mathrm{Cu}(110)$, Oxygen adsorption, Added-row reconstruction, Structural relaxation

Received:March 15, 2005; Revised:April 8, $2005 . \quad$ Correspondent:TAN, Ming-Qiu(E-mail:mqtan@zju.edu.cn; Tel/Fax:0571-87951328). "The Project Supported by NSFC(10204018) 